\title{
The Ultrasound File Format (UFF) - First draft
}

\author{
Olivier Bernard * \\ CREATIS \\ University of Lyon, \\ Lyon, France \\ Arun Nair \\ PULSE Lab \\ Johns Hopkins University \\ Baltimore, United States \\ David Bradway \\ Dept. of Biomedical Engineering \\ Duke University \\ Durham, United States
Dimitris Perdios
EPFL \\ Signal Processing Laboratory \\ Lausanne, Switzerland
}

\author{
Hendrik H.G. Hansen \\ Medical UltraSound Imaging Center \\ Radboud University Medical Center \\ Nijmegen, Netherlands
}

Stefano Ricci

Engineering Information Dept.

University of Florence

Florence, Italy

\author{
Pieter Kruizinga \\ Biomedical Engineering \\ Erasmus MC \\ Rotterdam, Netherlands \\ Ole Marius Hoel Rindal \\ Dept. of Informatics \\ University of Oslo \\ Oslo, Norway
}

\author{
Alfonso Rodriguez-Molares \\ Dept. Circulation and Medical Imaging \\ NTNU \\ Trondheim, Norway
}

\author{
Matthias Bo Stuart \\ Biomedical Engineering \\ Technical University of Denmark \\ Lyngby, Denmark
}

\author{
Pedro Filipe Viseu Dos Santos \\ Dept. of Cardiovascular Imaging and Dynamics \\ KU Leuven \\ Leuven, Belgium
}

\begin{abstract}
The lack of a standard format for storing ultrasound research data is hindering our ability to share and compare research results. This is slowing down the progress in our field, making it difficult to assess the relevance of new techniques.

In October 2017 the Ultrasound File Format (UFF) initiative was formed with the aim of defining such a standard, with the support of eleven research groups. Here we present some of the components of the first draft of the UFF format.

Index Terms-ultrasound, file, format, channel data, data sharing, research data, data reuse
\end{abstract}

\section{INTRODUCTION}

Systems able to produce channel data have become widely available to research laboratories, enabling the development of advanced sequences and processing techniques.

Despite its popularity, channel data are often stored in formats defined by the laboratories themselves, meeting their particular interests and tools. Those formats are often highly efficient, but they are fitted to specific sequences, systems, or probes, omitting information that would be required by a third party to use the data. They are difficult to maintain and expand.

Due to the increasing complexity of ultrasound imaging techniques, it no longer suffices with a set of images to assess the validity, correctness, and relevance of new techniques [1]. In recent years, there has been an increase in willingness to embrace data sharing practices [2]. Over $63 \%$ of researchers submit research data files as supplementary information for their manuscripts, or deposit the files in an open repository. It has been shown [2] that this practice increases the return on investment, enabling the reproduction, assessment, and reuse of research.

However, in the field of ultrasound imaging, the prevalence of custom formats hampers the diffusion of data.

In the occasion of the IEEE IUS 2017 conference (Washington D.C), the Ultrasound File Format (UFF) initiative

\footnotetext{
*Authors are shown in alphabetical order.
}

was started. Eleven laboratories agreed upon the need for a common format for channel data that would facilitate dissemination of data, replication of results, and comparison of processing techniques.

And hence the UFF taskforce was formed, composed by one member from each laboratory, with the objective of developing the common format. During most of 2018 the UFF taskforce has worked in the first draft of the standard. A draft that is disclosed in the following sections.

\section{Methods}

All units are given in SI units unless otherwise specified. We use blue color to identify references to UFF objects, and green to identify arrays of UFF objects.

Hierarchical Data Format (HDF5) was chosen due to its ability to store and organize large amounts of data [3]. HDF5 is a platform independent data model and file format that supports an unlimited variety of datatypes. It is designed for flexible and efficient I/O in local and remote systems.

HDF5 is developed by the HDF Group, a non-profit organization with the mission of advancing state-of-the-art open source data management technologies. HDF5 has become a de facto standard in the scientific community. Many scientific environments count with a HDF5 API such as MATLAB [4], Python [5], or Julia [6].

HDF5 uses two objects types: groups and datasets. HDF5 groups organize data objects similarly to directories and files in UNIX. Every HDF5 file contains a root group that can contain other groups. HDF5 datasets contain data and metadata that describe the data [3].

We use the term UFF object to refer to an independent set of data. UFF objects are stored as HDF5 groups, and are suited to be implemented as classes in several programming languages. 

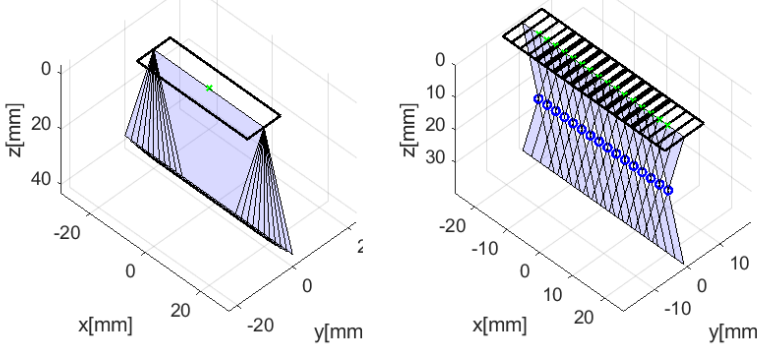
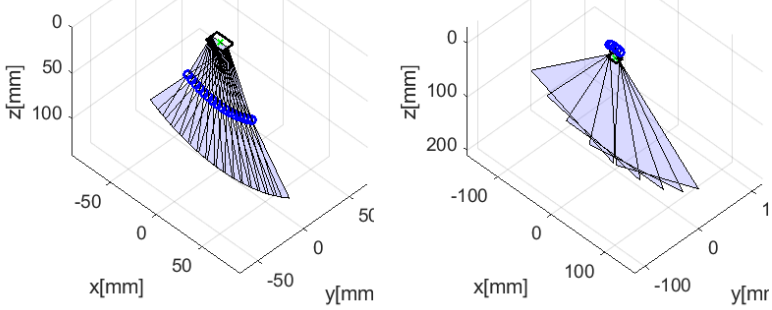

Fig. 1: Example of sequences

\section{RESULTS}

In this section we describe the objects that have been included in the first draft of the UFF.

\section{A. Channel data}

The object uff.channel_data contains all the information needed to store and later process channel data,

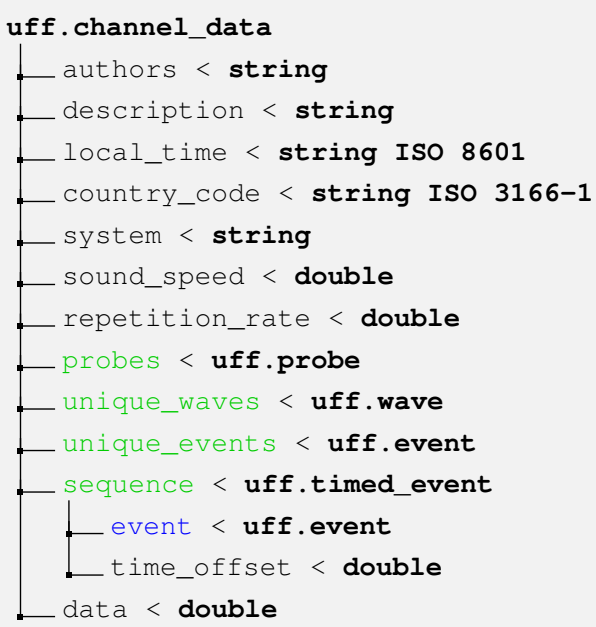

where authors identify the authors of the data; description describes the acquisition scheme, motivation and application; local_time and country_code identify the time and place the data were acquired; system describes the hardware used in the acquisition; sound_speed contains the reference speed of sound that was used in the system to produce the transmitted waves; and repetition_rate is the sequence repetition rate, also referred to as framerate in some scenarios

The object uff.channel_data contains all the probes used in the acquisition, a list of the unique_waves that have been transmitted, and a list of the unique_events that form the sequence. The sequence is specified as an array of uff.timed_events, each member containing an event reference, and the time_offset since the beginning of the beginning of the current repetition, also referred to as frame.

The HDF5 dataset data contains the channel data, organized as a matrix of four dimensions: samples $\times$ channels $\times$ events $\times$ repetitions

where samples is the number of temporal samples acquired by the system, channels is the number of active channels, events is the number of events in the sequence (not unique events), and repetitions is the number of times the sequence was repeated.

This proposal has the limitation of requiring that all event acquisitions have the same number of time samples and active channels.

\section{B. Events}

We define uff.event as a tuple of uff.transmit_setup and uff.receive_setup objects,

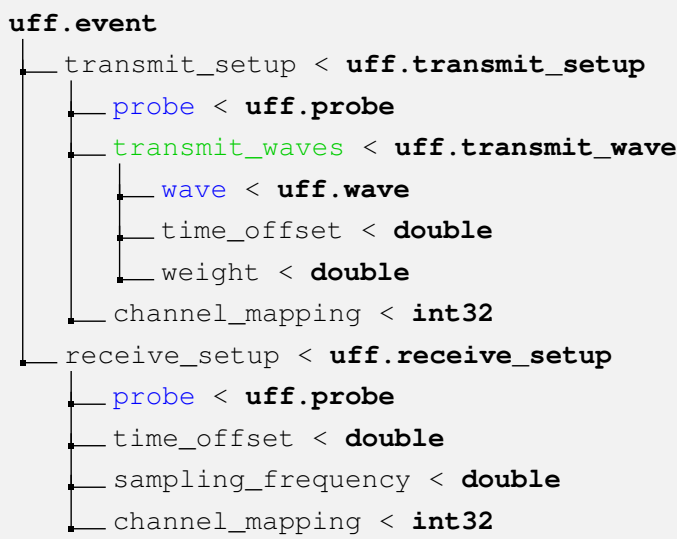

The object transmit_setup contains a reference to the probe used to transmit the waves, an array describing all the transmit_waves, and a channel_mapping dataset routing the elements in the probe to the active channels in the system.

Each of the transmit_waves contains a reference to a wave, and the time_offset since the start of the event. Note that several waves can be transmitted simultaneously, with different geometry, weight and time_offset relative to each other.

The object receive_setup contains a reference to the probe used on receive, the time_offset between the event start and the beginning of data acquisition, the sampling_frequency, and a channel_mapping dataset routing the elements in the probe to the channels in the system. 
The geometry of an ultrasonic wave is defined as

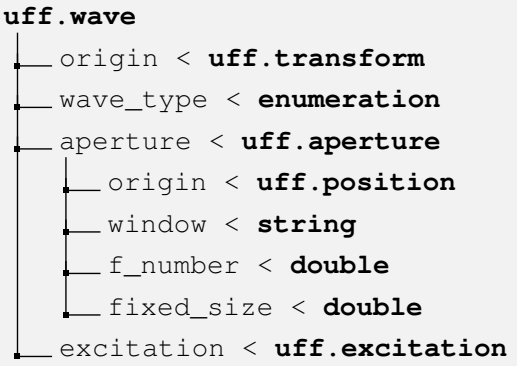

where wave_type is an enumerated type (int32) that indicates the type of transmitted wave: converging wave (0), diverging wave (1), plane wave (2), or cylindrical wave (3). The interpretation of origin depends on wave_type. For converging waves origin.translation holds the location of the wave focal point, for diverging waves origin.translation holds the location of the virtual source; in both cases the origin.rotation is ignored. For plane waves origin.rotation holds the orientation of the wave and origin.translation is ignored.

While origin completely defines the transmit delay profile, the aperture defines the transmit apodization profile. In this case aperture.origin defines the center of the aperture. The size of the aperture can be described with fixed_size, or with f_number in which case the aperture size is $A=d / F$ where $F$ is the f_number and $d$ is the distance between uff.wave_geometry.origin and uff.wave_geometry. aperture.origin.

The object window is a string describing the apodization window. Examples of uff.wave are shown in Fig. 2

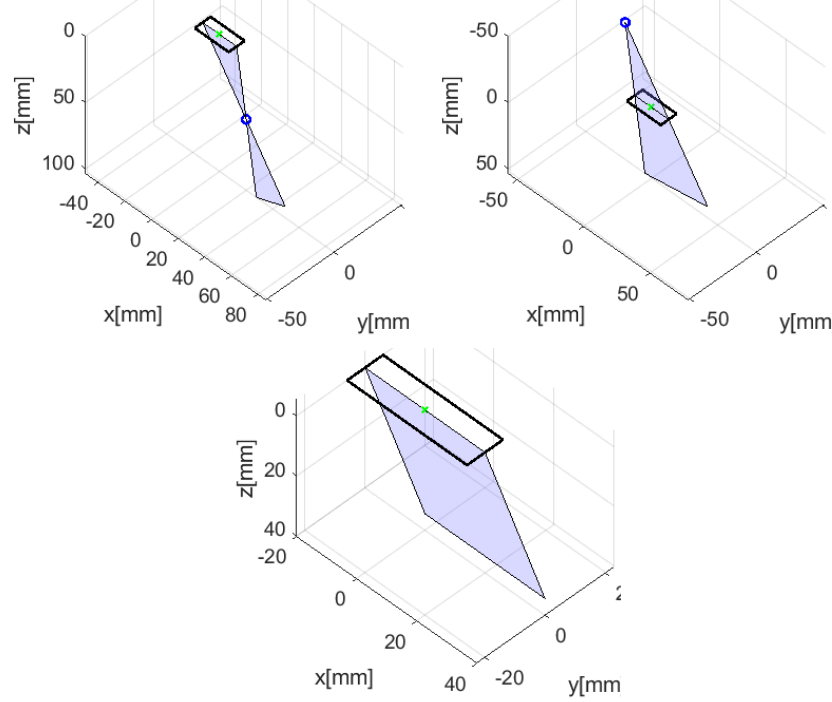

Fig. 2: Example of waves defined with uff.wave
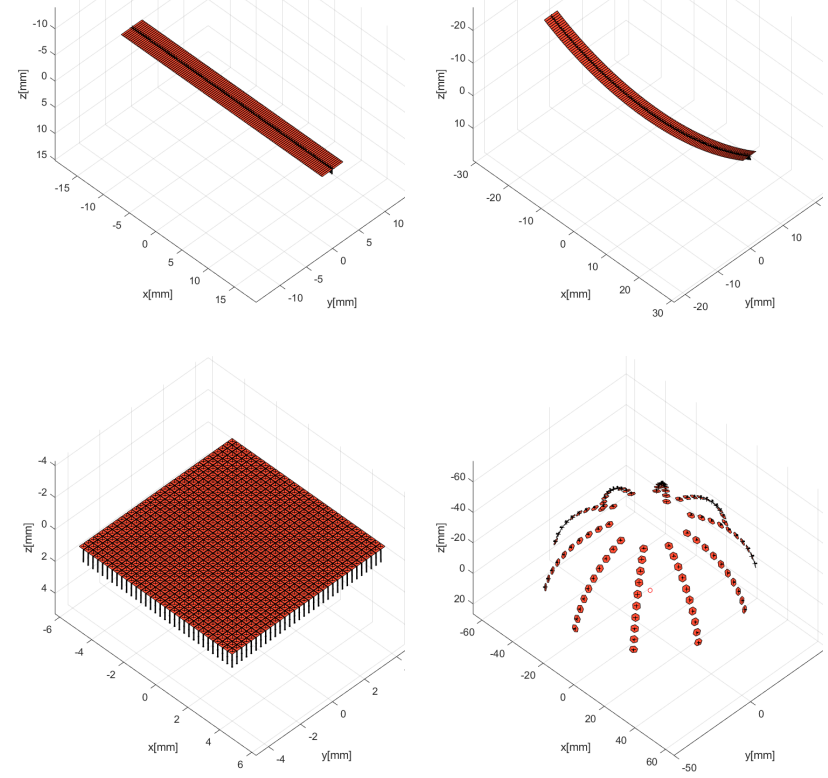

Fig. 3: Example of probes defined with uff.probe

\section{Probes}

UFF aims to support all possible probe geometries by defining uff.probe as an arbitrary collection of elements

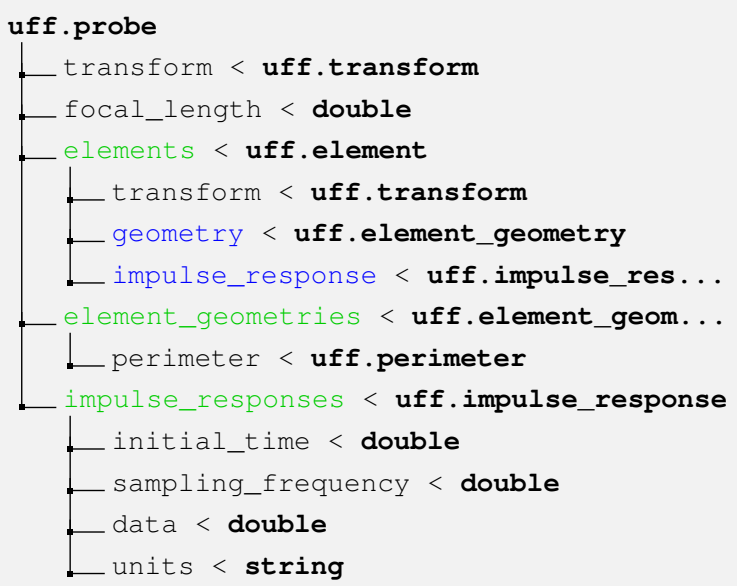

where focal_length specifies the lens focusing distance. Note that the elements in element_geometry and impulse_response are referred by the fields geometry and impulse_response of each member in element. This avoids unnecessary replication of information.

The objects in elements describe an ultrasonic element with a given geometry and impulse response, located at a given location in space. The objects in element_geometry define the geometry of the elements that have unique geometry (i.e. in a linear array element_geometry will have size 1). 


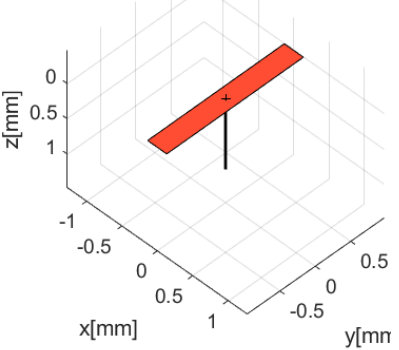

(a)

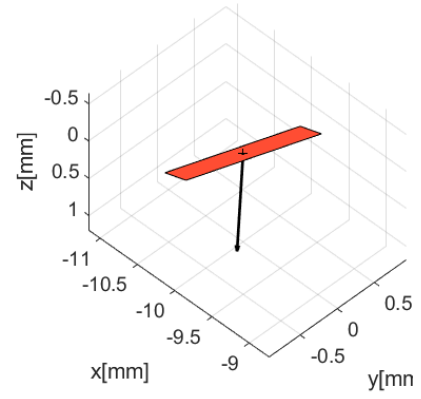

(c)

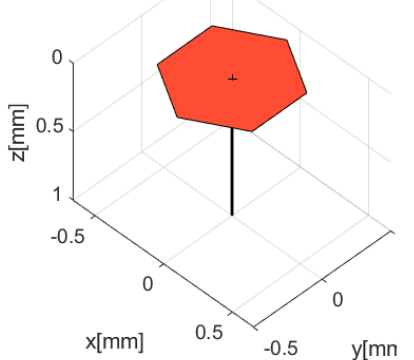

(b)

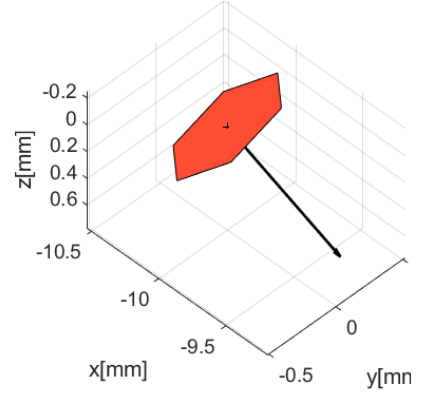

(d)
Fig. 4: Example of element geometries (a and b) and corresponding elements after transformation ( $c$ and $d$ )

Here we assume that the acoustic center of the element is at origin $O=(0,0,0)$ pointing towards $\vec{z}=(0,0,1)$. The element shape is defined by a closed perimeter contained within the $X Y$-plane, that is in turn composed of an ordered set of uff.position instances. In Fig. 4 examples are shown of two different element geometries and the attitude they take after applying the transform in the corresponding element.

The objects in impulse_responses describe the the two-way impulse response of unique elements, where initial_time denotes the time offset between the activation of the impulse and the first acquired sample. Note that geometry and impulse_response are references (H5Link).

The probe contains a uff.transform object that enables moving the probe in space, a convenient feature in probe tracking applications, or if more than one probe is used.

\section{E. Transforms}

The object uff.transform, which is included in many UFF objects, defines an affine transformation in a 3D Cartesian system, as

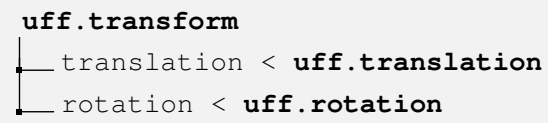

A transform is composed by a rotation $R=\left(r_{x}, r_{y}, r_{z}\right)$ followed by a translation $T=\left(t_{x}, t_{y}, t_{z}\right)$. The rotation axis $\mathrm{X}$, depicted in Fig. 5, is known as elevation, the rotation axis $\mathrm{Y}$ is known as azimuth, and the rotation axis $\mathrm{Z}$ is known as roll, in aeronautical terminology.

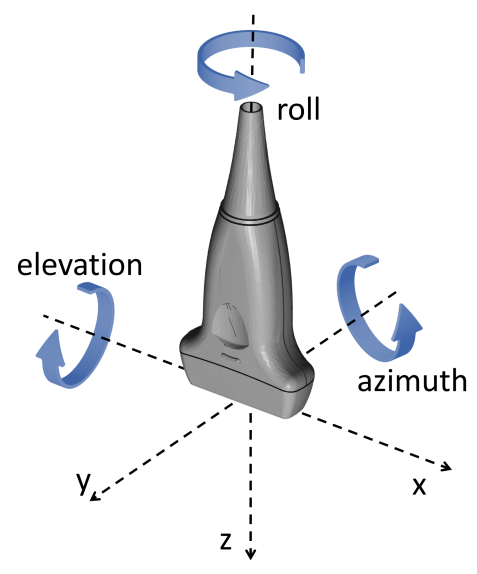

Fig. 5: 3D Cartesian axes and rotations.

\section{CONCLUSION}

We present here the main components in the first draft of the Ultrasound File Format (UFF). The scope of this first draft is limited to the specification of the components needed to store and process channel data. The UFF aims to be as general as possible, covering all possible probes, systems, and transmission schemes.

The format is based on HDF5, a platform independent file format designed for efficient handling of large amounts of data.

UFF is developed by the UFF taskforce, a group of researchers aiming to facilitate and popularize the dissemination of research data, replication of results, and comparison of processing techniques.

At the moment of this publication, the first draft is being discussed and revised by the UFF taskforce. However files and code examples are available at https://bitbucket.org/ ultrasound file format/uff/wiki/Home.

\section{ACKNOWLEDGMENT}

The UFF taskforce would like to thank the IEEE IUS organizing committee for providing a venue for UFF meetings, and our home institutions for supporting our activity in the UFF initiative.

\section{REFERENCES}

[1] O. M. H. Rindal, A. Austeng, H. Torp, S. Holm, and A. RodriguezMolares, "The dynamic range of adaptive beamformers," in 2016 IEEE International Ultrasonics Symposium (IUS), pp. 1-4, Sept 2016.

[2] N. A. Vasilevsky, J. Minnier, M. A. Haendel, and R. E. Champieux, "Reproducible and reusable research: are journal data sharing policies meeting the mark?," PeerJ, vol. 5, p. e3208, 2017.

[3] The HDF Group, "Introduction to hdf5." https://portal.hdfgroup.org/ display/HDF5/Introduction+to+HDF5 2018. [Online; accessed 2018-0917]

[4] Mathworks, "Hdf5 files." https://www.mathworks.com/help/matlab/ hdf5-files.html?searchHighlight=hdf5\&s_tid=doc_srchtitle 2018. [Online; accessed 2018-09-17].

[5] A. Collette, "Hdf5 for python." http://docs.h5py.org/en/stable/. 2018. [Online; accessed 2018-09-17].

[6] T. Holy, "Julia hdf5 guide." https://github.com/JuliaIO/HDF5.jl/blob/ master/doc/hdf5.md 2018. [Online; accessed 2018-09-17]. 